\title{
Les équipements de contrôle-commande des ouvrages hydrauliques d'Electricité de France
}

\author{
Jacques LECOUTURIER \\ Service de la Production hydraulique \\ Electricité de France
}

A la création d'Electricité de France (EDF) en 1946, le service de la Production hydraulique (SPH) prenait en charge 311 centrales, dont 19 seulement étaient partiellement automatisées et exploitées sans personnel permanent de conduite.

Aujourd'hui, le SPH exploite 470 centrales hydroélectriques, la puissance installee est de $22900 \mathrm{MW}$, tandis que la productibilité en année moyenne correspond à $63000 \mathrm{GWh}$.

Pour conduire ces 470 centrales, il n'existait plus en 1989 que dix-neuf services permanents de conduite, répartis en onze services locaux dans les centrales et huit dans les postes centralisés de conduite. A la fin de 1990, ce nombre sera ramené à quatorze avec respectivement trois services locaux et onze dans des postes centralisés. Quand un aménagement nouveau est construit, il est, dès le départ, conçu pour être automatique.

A l'horizon 2000, toutes les centrales hydroélectriques seront automatiques, quelle que soit la complexité des ouvrages, et moins de 10 postes centralisés seront nécessaires.
Cette évolution a été progressive, elle a passé par plusieurs stades successifs :

- commande manuelle actionneur par actionneur ;

- commande centralisée locale ;

- semi-automatique avec gardiennage ;

- automatique autonome ou téléconduite ;

pour arriver maintenant aux ensembles de centrales téléconduites ou téléprogrammées depuis un poste central de "poste hydraulique de vallée..." (PHV).

En cas de défaut nécessitant une intervention, le personnel d'exploitation est prévenu à son domicile par des messages téléphonés lui indiquant la nature et le lieu du défaut.

Parallèlement, dans le système Production-Transport d'EDF, le rôle des centrales hydroélectriques s'est modifié au cours des années. Jadis source principale, l'énergie produite par ces centrales représente maintenant $20 \%$ de la production nationale. Dans ce système à forte prédominance nucléaire, l'hydraulique joue un rôle essentiel par ses qualités dynamiques, sa disponibilité, sa rapidité de réaction et sa souplesse de réglage $(2000 \mathrm{MW}$ en $5 \mathrm{~min}$ pour la chaîne de la Durance).

Automatic control system of hydroelectric power stations

In a mainly nuclear production system, hydroelectric power stations are no longer used as the main source of electrical energy but, in a large extent, they allow dynamic performances to the electrical network with their availability, flexibility and rapidity.

At present, EDF operates hydroelectric power plants, most of them with automatic remote control and no local operator. The main power plants are operated from a Valley hydraulic station.

Renovation of the control systems of large plants will be necessary during the coming years. It will take place as a part of the automatic control of generating and transmission system and the implementation of new dispatchings of energy (CASOAR project). 


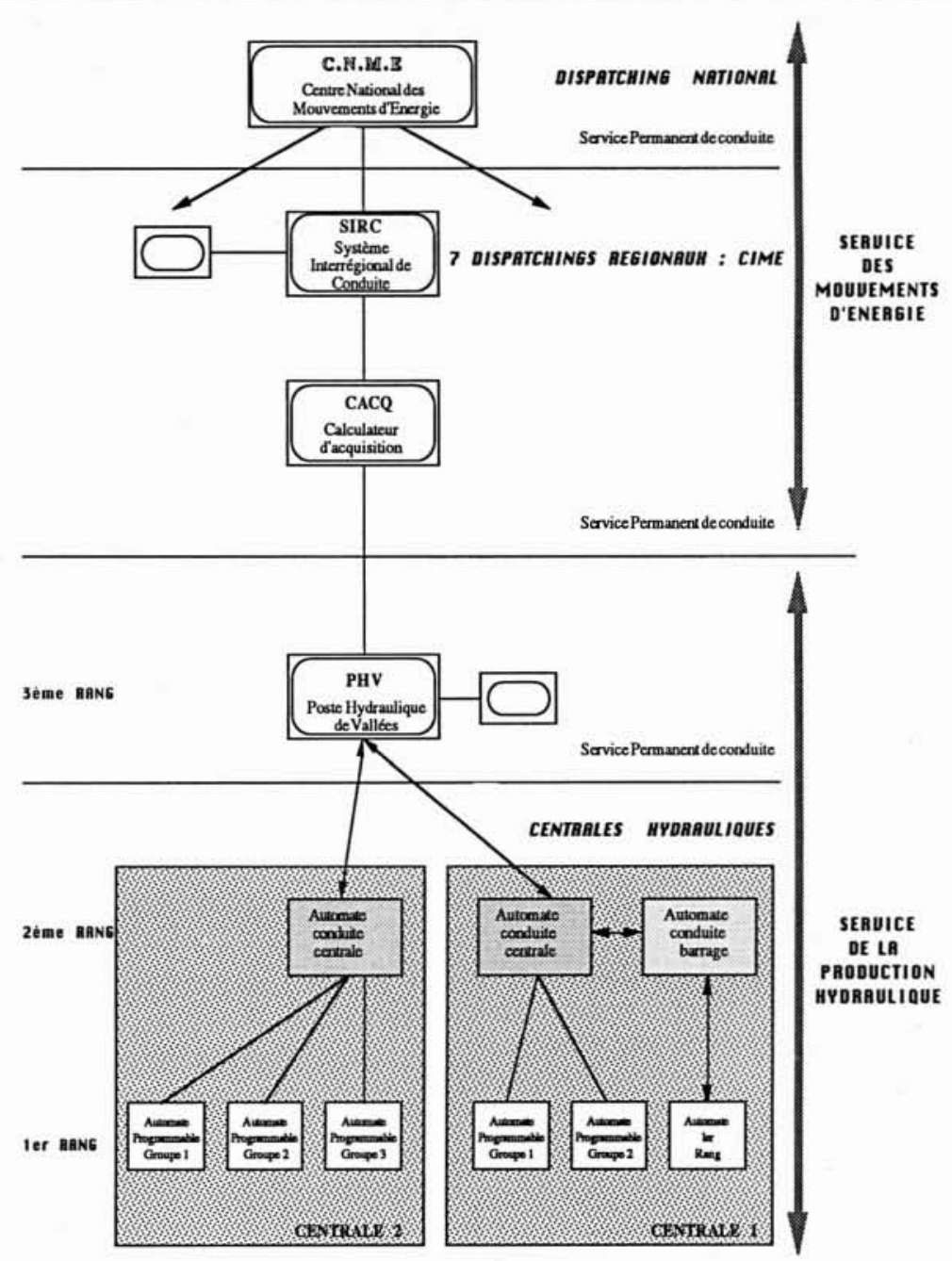

1. Architecture de conduite

\section{Principes actuels de conduite}

Le système de conduite des installations de production hydraulique est un sous-ensemble de celui de la Production-Transport, qui est géré aujourd'hui depuis les sept dispatchings des centres interrégionaux des Mouvements d'Energie coordonnés par le centre national des Mouvements d'Energie (CNME) ou dispatching national (fig. 1).

Chaque dispatching régional est équipé d'un double ensemble informatique : l'un d'acquisition (CACQ), l'autre de conduite (SIRC).

Dans le cas des aménagements de production hydraulique, le dispatching régional reçoit des informations venant des centrales et, par la même liaison, il transmet ses consignes de marche (programmes) à celles-ci. Tous ces échanges sont effectués par l'intermédiaire du poste central du PHV, dont une des fonctions principales est la centralisation de la conduite en temps réel des centrales.

\section{Architecture de la conduite}

Depuis de nombreuses années, le service de la production hydraulique a développé des moyens de conduite automatique de ses centrales pour exploiter au mieux leur souplesse d'intervention et répondre dans les délais les plus courts aux demandes des dispatchings. Cette conduite automatique est conçue selon un système hiérarchisé à trois niveaux (ou rangs) d'automates, qui sont représentés sur la figure 1 .

L'automate de premier rang est en liaison directe avec le matériel électromécanique, groupe turbo-alternateur, vanne d'évacuateur de crues.

L'automate de second rang est situé à la centrale ou au barrage, dont il assure la conduite globale ainsi que la surveillance.

L'automate de troisième rang est constitué par le poste central du PHV ; il est à la fois l'interlocuteur du dispatching régional et des automates de second rang des centrales. 


\section{Classification des centrales}

Le parc de centrales à conduire est très hétérogène, tant par sa nature que par le service à rendre :

— en puissance installée (de $60 \mathrm{~kW}$ à $1800 \mathrm{MW}$ );

- en hauteur de chute (quelques mètres à $1400 \mathrm{~m}$ );

— en débit transité (quelques $\mathrm{m}^{3} / \mathrm{s}$ à plus de $1000 \mathrm{~m}^{3} / \mathrm{s}$ );

— en valeur de placement d'énergie : fil de l'eau, éclusée, lac, transfert par pompage.

Toutes les petites centrales ont un fonctionnement automatique assuré par leur automate de second rang; leur surveillance est effectuée par le personnel d'une centrale plus importante.

La conduite des autres centrales est réalisée en distinguant trois catégories:

\section{- Les centrales téléconduites}

Elles comportent les centrales de lac, des stations de transfert d'énergie par pompage et des centrales enchaînées le long d'un même fleuve : elles sont aussi dénommées " centrales d'importance nationale ». Elles ont une influence importante dans l'exploitation du système productiontransport : capacité de production, vitesse de modulation et procédure de sauvegarde du réseau.

Pour ces centrales, le système PHV autorise les actions les plus rapides sur les groupes : 5 à $6 \mathrm{~s}$ pour modifier une consigne.

\section{- Les centrales téléprogrammées}

Ces centrales "régionales" ont individuellement moins d'influence (puissance installée et capacité de modulation faibles) ; la téléprogrammation permet une optimisation de leur gestion économique sans faire intervenir les exploitants locaux. En outre, en cas de situation tendue sur le réseau, elles peuvent recevoir un ordre de passage à puissance maximale.

\section{- Les centrales télésurveillées}

Ces centrales, souvent conduites au fil de l'eau, ont peu d'influence dans l'exploitation du système production transport : elles sont conduites de façon autonome par leur automate de second rang. La télésurveillance permet la supervision de leur fonctionnement ainsi que la fourniture des informations hydrauliques nécessaires, soit pour la conduite de centrales plus importantes, soit pour l'information des tiers.

\section{Principes, fonctions et matériels actuels}

\section{Les automates de premier rang}

Ils assurent les fonctions de sécurité des installations et l'enchaînement des actionneurs pour réaliser une fonction, par exemple : démarrage/arrêt d'un groupe ou régulation de niveau d'un puits d'exhaure.
La majorité des automates en exploitation sont encore à logique câblée, au moyen de relais électromagnétiques (environ $85 \%$ du parc). Ils ont été réalisés dans les années 70 , selon une schématèque type définie pour l'ensemble du $\mathrm{SPH}$. Les principes retenus et rappelés ci-après sont toujours valables, même si la technologie a changé :

- le fonctionnement normal est automatique ;

- la tension de contrôle-commande est fournie par une batterie unique et distribuée selon une tranche continue par groupe (125 V = à l'époque);

- l'état de sécurité d'un groupe est l'arrêt ;

- l'état de sécurité d'une centrale dépend des conditions hydrauliques ;

— les schémas sont à "manque de tension ", c'est-à-dire que la disparition de la tension de contrôle provoque le retour à l'état de sécurité : l'arrêt ;

- les schémas sont exécutés avec des hypothèses simplificatrices ;

Les automates programmables ont commencé à être mis en place à partir de 1978, sous deux versions :

- automates programmables industriels (API) sur les groupes de puissance unitaire inférieure à $30 \mathrm{MW}$;

- automates programmables spécifiques à traitements redondants pour les grosses unités (Grand'Maison, Montezic, Haut Rhone).

Un recueil de " grafcets types » a été établi pour remplacer la schématèque à relais.

\section{Les automates de second rang}

Ils assurent le fonctionnement automatique des installations selon les divers cas d'exploitation, en utilisant les automatismes de premier rang. Les fonctions réalisées sont notamment :

- la gestion des liaisons de télétransmissions avec l'automate de $3^{\mathrm{e}}$ rang;

- l'application des consignes reçues par l'automate de $3^{\mathrm{C}}$ rang ;

- la régulation de niveau de la retenue;

- la répartition des consignes sur les groupes et les vannes ;

- l'application des consignes de crues pour un barrage ; - la transmission d'informations vers l'automate de $3^{\mathrm{e}}$ rang ;

- la tenue du journal de bord de la conduite.

Les plus anciens sont analogiques; maintenant, selon l'importance de la centrale, ce sont soit des automates programmables industriels, soit des minicalculateurs ou une association des deux dans les cas complexes. Le parc actuellement en exploitation comporte 360 automates programmables industriels en $1^{\text {er }}$ et $2^{\mathrm{e}}$ rang et une centaine de minicalculateurs.

\section{Les automates de troisième rang}

Ils remplissent les fonctions nécessaires à la conduite des postes de commande centralisée (aujourd'hui PC de PHV). 
Le rôle des automates de troisième rang est triple:

- Pour le réseau, ils exécutent les ordres venant du dispatching et renseignent le dispatcheur régional pour faire face à trois types de situations :

- normale : optimisation économique de la production ;

- perturbée : mise en œuvre rapide des actions nécessaires pour faire face aux défaillances;

— incident généralisé : remise sous tension du réseau par les groupes hydrauliques.

- Surveillance et coordination des écoulements de débit et des aménagements :

- fonctionnement des centrales enchaînées sur un même fleuve ;

- suivi global et aide au passage des crues;

- alerte dans les cas graves.

- Concentration d'informations pour le Service des Mouvements d'Energie et pour les tiers.

Les premières réalisations ont été mises en service en 1975. A cette époque, les rôles vis-à-vis du réseau et la surveillance des écoulements étaient assurés par des postes différents, mais l'évolution entreprise en 1980 avec le poste commun de conduite de SAINTE-TULLE a permis en 1984 de regrouper les rôles ci-dessus dans une même entité : les postes centraux de PHV.

En 1990, ce sont onze postes centralisés de conduite qui sont en service.

\section{Contrôle-commance des grandes centrales}

Jusqu'en 1985, les activités de rénovation d'équipements de contrôle-commande n'avaient pratiquement concerné que des centrales de moyenne importance. Compte tenu de leurs dates de mise en service (années 60), les grandes centrales exploitées par le SPH atteignent maintenant une période où il est nécessaire de rénover leurs automatismes. Un groupe de concepteurs du SPH a donc été chargé, en 1988, de définir les principales orientations à mettre en œuvre, en intégrant notamment l'expérience acquise depuis 1980 sur l'utilisation des automates programmables.

Les installations concernées sont les centrales équipées de groupes de production de puissance importante (supérieure à $30 \mathrm{MVA}$ ), y compris les stations de transfert d'énergie par pompage.

Les objectifs fixés sont :

- améliorer la sécurité sur le plan du matériel, mais surtout sur celui du logiciel d'application;

- maintenir une bonne disponibilité : moins d'une panne tous les 4 ans (performance actuelle des API);

- développer les aides à l'exploitation: maintenance, dépannage, diagnostic sur place ou à distance ;

- faciliter l'évolution des matériels.

\section{Les principales conclusions}

Pour faciliter les évolutions des matériels, il est nécessaire d'appliquer une définition rigoureuse des fonctions assurées par chaque rang d'automates, sachant que maintenant l'intelligence est répartie entre le premier et le second rang.

La solution retenue a les caractéristiques suivantes :

- emploi généralisé des automates programmables industriels (API) pour les automatismes de premier rang ;

- adjonction à l'automate principal (APG) de chaque groupe d'un automate de sauvegarde (APS), pour se prémunir contre les conséquences des défauts de matériel et logiciel ;

- utilisation de microcalculateurs de type compatible PC pour assurer les fonctions de consignation d'états et de traitement des informations ;

- introduction de réseaux locaux industriels hétérogènes, afin d'améliorer les échanges vers d'autres applications, mais aussi de permettre des évolutions indépendantes des différents niveaux d'automates;

- non concentration des automatismes des services auxiliaires généraux d'une centrale, dans un même équipement.

L'automate principal (APG) assure toutes les fonctions nécessaires à l'automatisme du groupe (séquentiel, traitement des protections, vraisemblance des états, exécution des actions de sécurité, application des consignes) et à la gestion des liaisons avec les réseaux locaux.

L'automate de sauvegarde (APS) n'empêche pas les ordres intempestifs éventuels, mais limite les conséquences de leur action. Dans ce but, il se comporte en observateur, d'une part du traitement correct des protections effectué par l'APG et, d'autre part, de la vraisemblance des états des organes de sécurité du groupe. En cas d'anomalie constatée, cet automate APS met le groupe à l'arrêt en coupant les polarités des actionneurs du groupe et signale en sortie le type de défaut (fig. 2).

En cas de défaut de l'automate principal (APG), le groupe est mis à l'arrêt, tandis qu'une défaillance de l'automate de sauvegarde (APS) ne provoque qu'une alarme.

Au niveau du réseau local, deux possibilités ont été conservées :

— un seul réseau à médium redondant véhiculant toutes les données ;

- un réseau pour la conduite, l'autre pour les informations.

Les premières études ont montré que la première solution était plus simple à mettre en œuvre et diminuait la charge des automates.

Selon le volume des informations nécessaires pour la consignation d'états, il peut être nécessaire d'installer à côté de l'automate de groupe, un second automate du même type, spécialisé dans l'acquisition et la datation (précision $20 \mathrm{~ms}$ pour les plus rapides). Ces deux possibilités sont représentées sur les figures 3 et 4 .

Les informations présentes sur le réseau peuvent aussi être utilisées pour alimenter les applications de maintenance du matériel électromécanique, d'aide au diagnostic et de surveillance locale ou à distance. 
PROTECTIONS

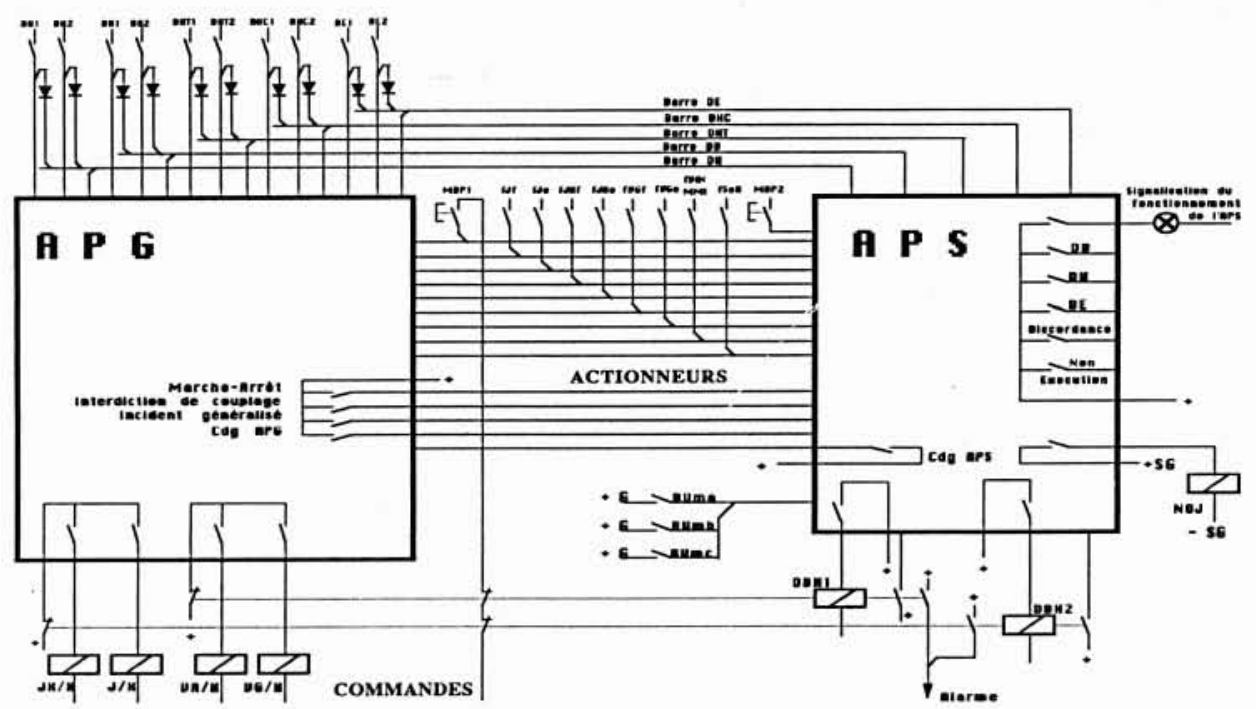

2. Contrôle-commande des grandes centrales - Automate de groupe.

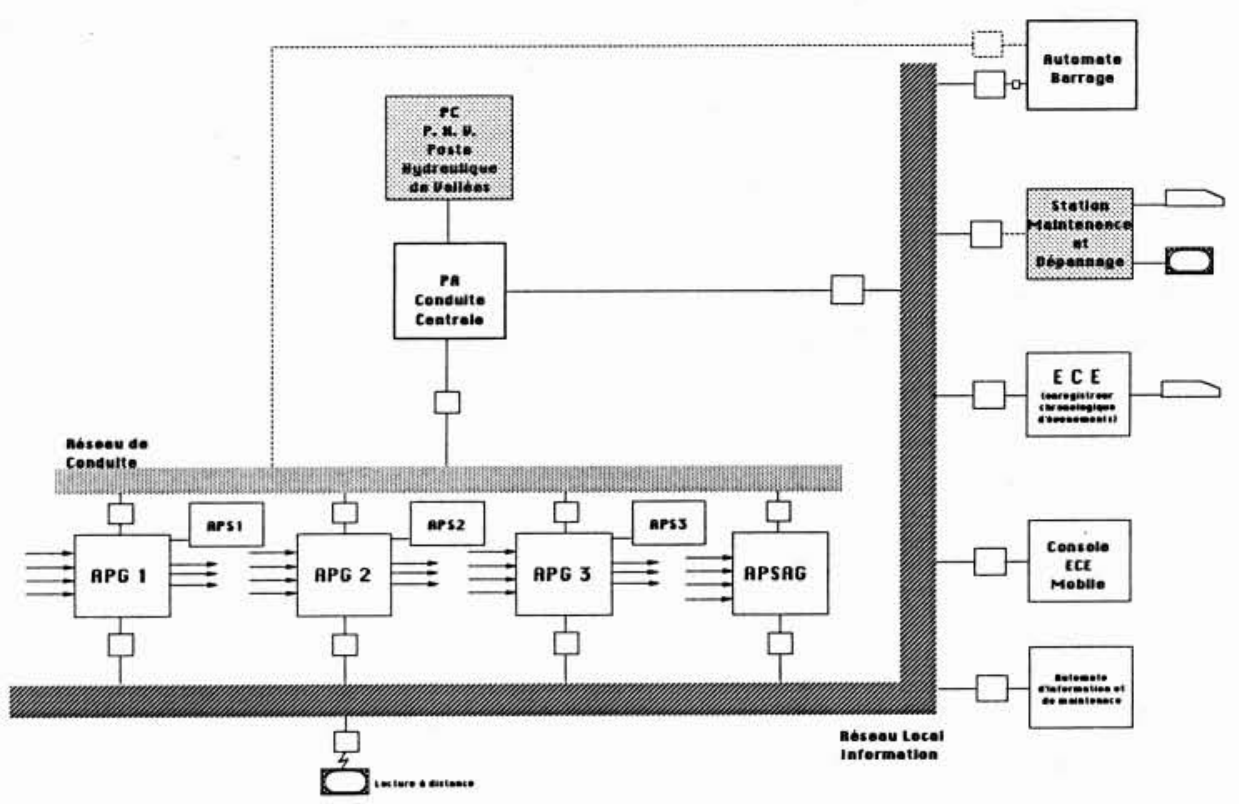

3. Contrôle-commande des grandes centrales - Solution avec deux réseaux séparés.

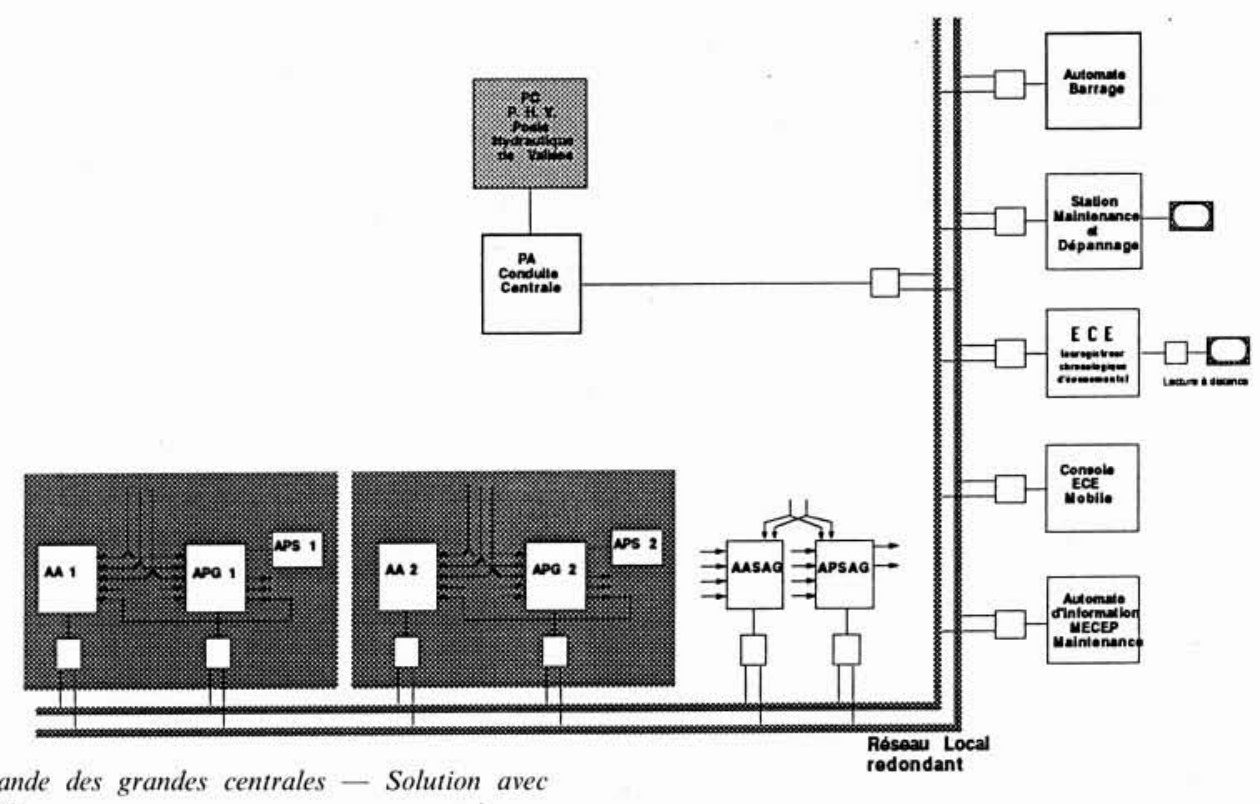

4. Contrôle-commande des grandes centrales - Solution avec réseau redondant. 


\section{Le nouveau système de conduite de la DPT}

La direction de la Production et du Transport d'EDF a décidé d'entreprendre la rénovation, à l'horizon 2000 , de ses dispatchings national et régionaux de conduite. Ce projet est dénommé «conduite automatique du système (DPT) optimisé en actif compte tenu du réseau» (CASOAR).

Actuellement le réglage automatique, pour obtenir l'égalité entre la puissance produite et celle consommée à la fréquence de $50 \mathrm{~Hz}$, est réalisé au moyen de deux régulations :

- Le réglage primaire effectué par les régulateurs de vitesse des groupes de production de manière à rétablir l'équilibre production-consommation à une fréquence différente de $50 \mathrm{~Hz}$ selon leur droite de statisme. Cette régulation a le temps de réponse le plus rapide, conditionné par la vitesse des organes d'admission (quelques dizaines de secondes pour les groupes hydrauliques),

- Le réglage secondaire ou téléréglage a pour mission de ramener la fréquence à $50 \mathrm{~Hz}$ en agissant sur les consignes de production des groupes, proportionnellement aux capacités de réglage de chacun. La bande de réglage sur le réseau français est en moyenne de $600 \mathrm{MW}$, dont généralement $75 \%$ fournis par les groupes hydrauliques.

Si la perturbation sur le réseau est importante, les marges de réglage ne sont plus suffisantes et il faut alors faire appel aux groupes en réserve : tournante pour les groupes thermiques, à l'arrêt pour les groupes hydrauliques, notamment les stations de transfert d'énergie par pompage. Cette réserve correspond à $4000 \mathrm{MW}$ environ, dont $80 \%$ d'hydraulique. Le choix des groupes et les consignes appliquées sont déterminés par les dispatcheurs selon les contraintes existantes et constituent le réglage tertiaire du réseau.

L'objectif des nouveaux systèmes de conduite de la direction de la Production et du Transport d'EDF est d'automatiser ce réglage tertiaire : chaque groupe recevra alors toutes les $10 \mathrm{~s}$ une télévaleur de consigne de puissance déterminée selon une optimisation nationale prenant en compte les contraintes de production du réseau de transport et les coûts de production. Cette télévaleur de consigne incluera la participation au réglage secondaire.

Compte tenu de la complexité de l'objectif à atteindre, le projet CASOAR a été échelonné en trois paliers :

- $I^{\text {er }}$ palier : remontée des informations vers les dispatchings (1994/1995);

$-2^{e}$ palier : mise à jour automatique des programmes de production en cours de journée ;

$-3^{e}$ palier : conduite automatique des groupes par envoi d'une consigne toutes les $10 \mathrm{~s}$.

La mise en place de ce système va également nécessiter la création, pour 1994, d'un nouveau réseau de téléconduite pour l'ensemble de la direction de la Production et du Transport, il s'agit du projet ARTERE mis en œuvre par le service du Transport et des Télécommunications.

\section{Centrales hydrauliques concernées}

Le premier palier de CASOAR se fixe done pour objectif de renseigner le système national de Conduite et les centres régionaux de Conduite sur l'état des centrales raccordées aux réseaux $400 \mathrm{kV}$ et $220 \mathrm{kV}$. Au total, la participation des centrales hydrauliques va concerner 137 centrales et 408 groupes de production.

Pour la gestion des centrales, trois catégories de données sont nécessaires :

- Les données fixes décrivent les caractéristiques des ouvrages, fournissent une description des centrales et des aménagements et sont contenues dans un fichier national. Ces données ne concernent pas les systèmes de conduite en temps réel.

- Les données prévisionnelles comportent les diagrammes de production envoyés par les dispatchings, les limitations de performances et les diagrammes prévisionnels de production à $15 \mathrm{~min}$ pour chaque groupe, fournis par les centrales. Ces données sont transmises par les équipements de conduite.

- Les données instantanées sont transmises en temps réel vers les dispatchings et constituent l'essentiel du premier palier de CASOAR. Ces données sont de deux catégories : d'une part, des signalisations d'états et des mesures filtrées prélevées sur les groupes, d'autre part des informations composées (marge de production, débits, hauteurs de chute).

Les informations envoyées par le dispatching sont des modifications instantanées de consignes de production.

\section{Mise en place du $1^{\text {er }}$ palier CASOAR}

La mise en place du $1^{\text {er }}$ palier de CASOAR nécessite l'adaptation des systèmes de conduite existants. Or ceux-ci sont de générations très différentes, puisqu'ils ont été mis en service de 1975 à maintenant. Il faut également souligner un problème important de migration puisque ces automates doivent demeurer opérationnels pendant leur adaptation.

Quatre cas de figures se rencontrent pour les centrales du SPH :

- Les centrales isolées ou conduites à partir des postes de commande de la génération 75 sont celles des Pyrénées ou actuellement reliées au poste de commande hydraulique de Brive. Les délais exigés pour la mise en service du $1^{\mathrm{er}}$ palier CASOAR ne permettent pas de lier cette opération avec celle d'un système PHV de seconde génération, qui sera réalisée pour le deuxième palier. Néanmoins, la structure matérielle installée en 1994 pourra être utilisée pour implanter les logiciels nécessaires à la conduite pour les paliers suivants.

L'architecture envisagée est celle déjà décrite pour les grande centrales, elle est donnée sur la figure 5 .

- Les centrales sur les postes centralisés de la génération 80 , c'est-à-dire Châteauneuf et Génissiat pour le Rhône et Sainte-Tulle pour la Durance. Ces équipements déjà anciens n'ont pas atteint leur fin de durée de vie, mais il 
n'est pas possible d'envisager de modifications importantes sans remettre en cause leur bon fonctionnement. Le principe retenu consiste à traiter les fonctionnalités demandées pour le $1^{\mathrm{er}}$ palier de CASOAR de manière indépendante. La liaison vers le réseau ARTERE s'effectue en interposant un système d'interface sur l'actuelle liaison vers les calculateurs du dispatching régional. L'architecture envisagée est représentée sur la figure 6 ; elle est semblable dans ses principes à la précédente et pourra remplacer les équipements actuels à l'arrivée du $2^{\mathrm{C}}$ palier de CASOAR.

gements et sont contenues dans un fichier national. Ces données ne concernent pas les systèmes de conduite en temps réel.

- Les données prévisionnelles comportent les diagrammes de production envoyés par les dispatchings, les limitations de performances et les diagrammes prévisionnels de production à $15 \mathrm{~min}$ pour chaque groupe, fournis par les centrales. Ces données sont transmises par les équipements de conduite.

- Les données instantanées sont transmises en temps réel vers les dispatchings et constituent l'essentiel du premier palier de CASOAR. Ces données sont de deux catégories : d'une part, des signalisations d'états et des mesures filtrées prélevées sur les groupes, d'autre part des informations composées (marge de production, débits, hauteurs de chute).

Les informations envoyées par le dispatching sont des modifications instantanées de consignes de production.

Mise en place du $1^{\mathrm{er}}$ palier CASOAR

La mise en place du $1^{\text {er }}$ palier de CASOAR nécessite l'adaptation des systèmes de conduite existants. Or ceux-ci sont de générations très différentes, puisqu'ils ont été mis en service de 1975 à maintenant. Il faut également souligner un problème important de migration puisque ces automates doivent demeurer opérationnels pendant leur adaptation.

Quatre cas de figures se rencontrent pour les centrales du SPH :

- Les centrales isolées ou conduites à partir des postes de commande de la génération 75 sont celles des Pyrénées ou actuellement reliées au poste de commande hydraulique de Brive. Les délais exigés pour la mise en service du $1^{\mathrm{er}}$ palier CASOAR ne permettent pas de lier cette opération avec celle d'un système PHV de seconde génération, qui sera réalisée pour le deuxième palier. Néanmoins, la structure matérielle installée en 1994 pourra être utilisée pour implanter les logiciels nécessaires à la conduite pour les paliers suivants.

L'architecture envisagée est celle déjà décrite pour les grande centrales, elle est donnée sur la figure 5 .

- Les centrales sur les postes centralisés de la génération 80, c'est-à-dire Châteauneuf et Génissiat pour le Rhône et Sainte-Tulle pour la Durance. Ces équipements déjà anciens n'ont pas atteint leur fin de durée de vie, mais il n'est pas possible d'envisager de modifications importantes sans remettre en cause leur bon fonctionnement. Le principe retenu consiste à traiter les fonctionnalités demandées pour le $1^{\text {er }}$ palier de CASOAR de manière indépendante.

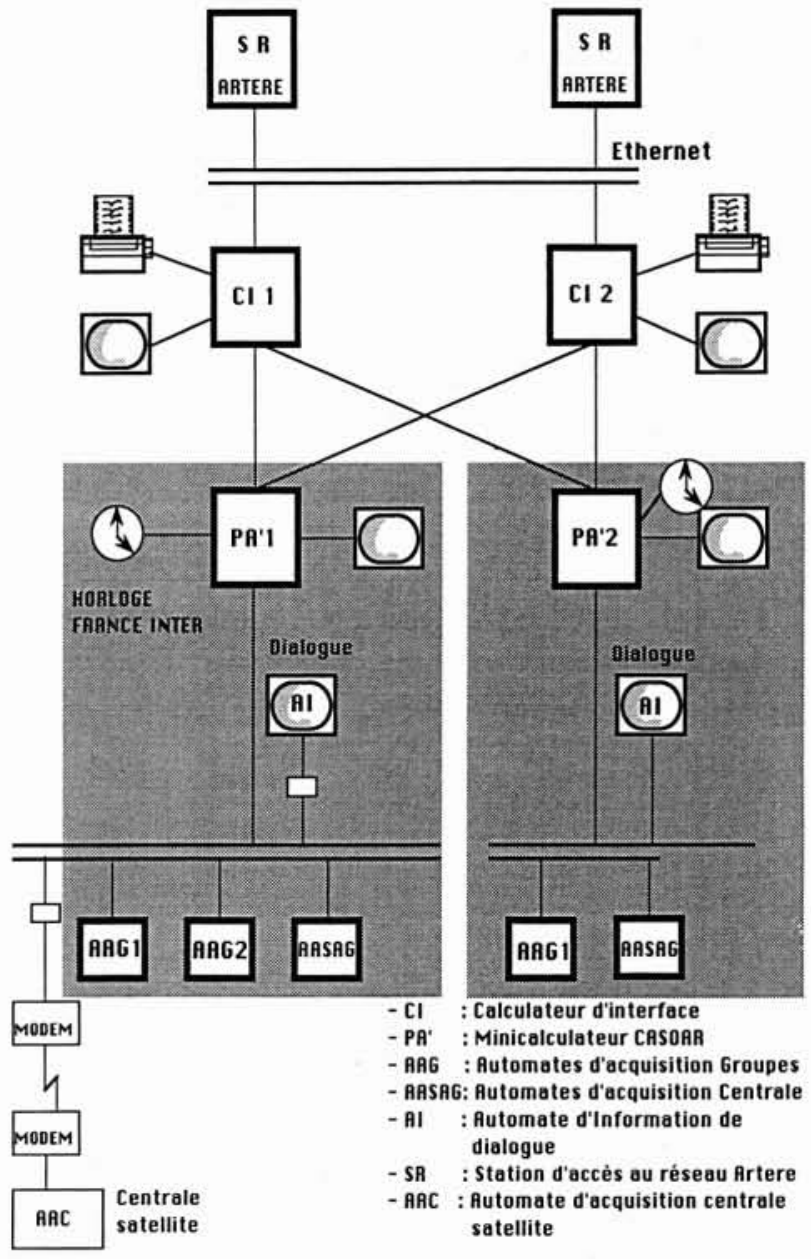

5. Architecture envisagée pour les postes de commande des centrales des Pyrénées ou reliées au PCH de Brive.

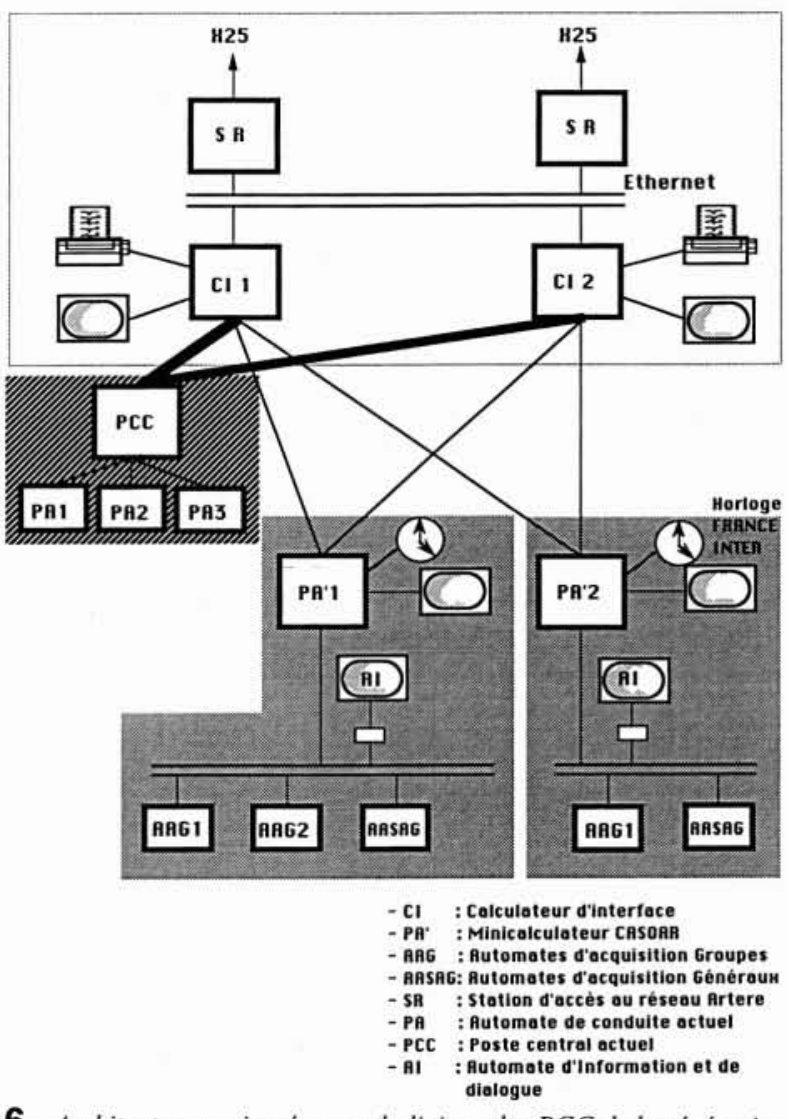

6. Architecture envisagée pour la liaison des PCC de la génération 1980 avec le réseau ARTERE. 
La liaison vers le réseau ARTERE s'effectue en interposant un système d'interface sur l'actuelle liaison vers les calculateurs du dispatching régional. L'architecture envisagée est représentée sur la figure 6 ; elle est semblable dans ses principes à la précédente et pourra remplacer les équipements actuels à l'arrivée du $2^{\mathrm{c}}$ palier de CASOAR.

- Les centrales des PHV de le génération sont celles des PHV Maurienne et du Rhin, elles vont être mises en service de 1990 à 1992. Pour éviter de multiplier le volume de matériel informatique, les fonctions du $1^{\mathrm{er}}$ palier CASOAR seront ajoutées aux logiciels actuels et la liaison vers le réseau ARTERE sera réalisée en interposant un système d'interface sur l'actuelle liaison vers les calculateurs du dispatching régional.

- Les centrales de la nouvelle génération de PHV sont celles actuellement conduites depuis le poste de Lyon dont les équipements seront renouvelés pour 1994. La configuration d'un tel poste central est réprésentée sur la figure 7 : il intègrera les fonctions, non seulement du premier palier, mais également celles du second. ${ }_{1}^{2}$

Les différentes fonctions du poste central sont traitées sur plusieurs processeurs redondants reliés par un réseau local industriel et spécialisés de la manière suivante: gestion des liaisons vers les centrales, traitements spécifiques du poste central, interface homme machine, station vers le réseau ARTERE.

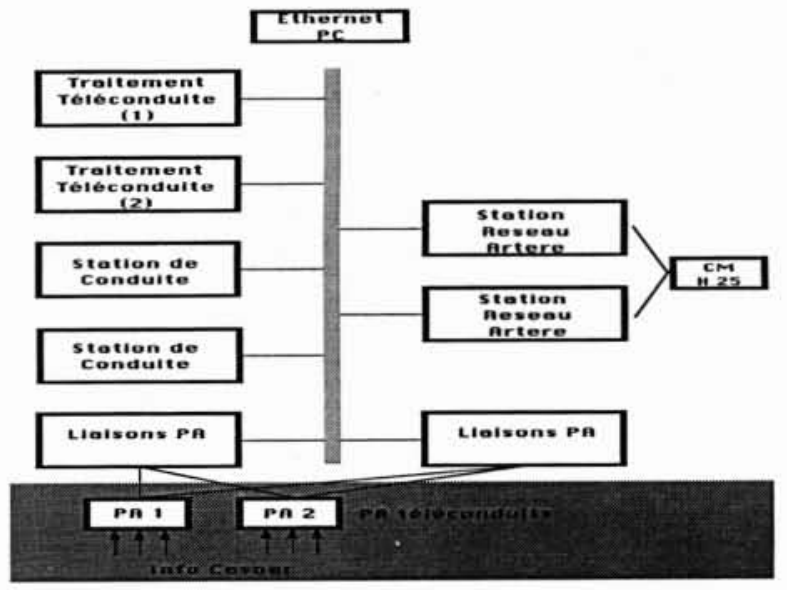

7. Configuration prévue pour le poste central d'un PHV de la nouvelle génération.

Le choix des matériels et des logiciels à grande diffusion et dont l'évolution est garantie devrait faciliter l'intégration des fonctionnalités du $3^{\mathrm{e}}$ palier de CASOAR.

\section{Conclusion}

A l'heure où l'équipement de nouveaux sites hydrauliques subit un ralentissement très net, pour diverses raisons : économiques et une certaine limitation du nombre des sites équipables, les perspectives en matière d'amélioration de la téléconduite et de la télésurveillance des centrales hydrauliques sont encore très vastes. Que ce soit pour les opérations de renouvellements des équipements de contrôle-commande des centrales ou pour l'implantation des fonctions demandées pour le nouveau système de conduite de la direction de la Production et du Transport d'EDF, les équipes de maitrise d'œuvre du service de la
Production Hydraulique ont un volume très important d'applications à réaliser d'ici l'an 2000.

L'utilisation de matériels standards du marché ainsi que de produits logiciels généraux devraient faciliter la réalisation des nouveaux équipements et améliorer leur évolutivité. Néanmoins, il ne faut pas perdre de vue que les centrales hydrauliques sont des processus simples: la simplification des automatismes mis en œuvre par nos prédécesseurs depuis maintenant plus de 20 ans, doit rester la règle. La sophistication des équipements de contrôlecommande ne va pas de pair, ni avec la fiabilité, ni avec la disponibilité des ouvrages qui permettent d'obtenir les qualités dynamiques exigées par le réseau.

\section{Bibliographie}

HiPPERT M. - Evolution des techniques de conduite et de surveillance dans les usines hydroélectriques RGE Mars 1978.

Lecouturier J., Weisrock G. - Les Postes Hydrauliques de Vallées. ONU. Séminaire sur la comparaison des modèles de planification et d'exploitation des systèmes électriques. Moscou. Juin 1987.
Germain P., Lecouturier J. - Valley hydraulic Stations Symposium IFAC On the application of system analyses to water management. Rabat. Octobre 1988.

LAVy P., LecouturieR J. - JP Katz: Les automates de contrôle des aménagements hydroélectriques. Revue de l'Energie. Mars-Avril 1989.

Mourier M., Lecouturier J., Digue C., Piat H. - Automation of the La Rance Tidal Power Plant. Conférence Water Power. Uprating and refurbishing hydro power plants. Zurich. Octobre 1989. 